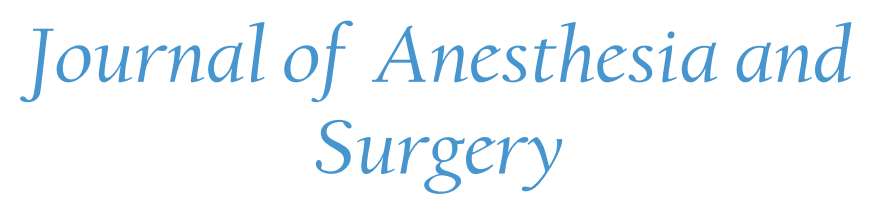

\title{
Pseudoaneurysm of Mitral-Aortic Intervalvular Fibrosa Following Regurgitation of Congenital Bicuspid Aortic Valve in a 63-Year-Old Patient: A Case Report
}

\author{
Li Zhou ${ }^{1 \#}$, Hong Chang ${ }^{1,2 \#}$, Yi Yang ${ }^{2}$, Wei Cao ${ }^{2}$, Chunling Jiang ${ }^{*}$ \\ ${ }^{1}$ Department of Anesthesiology, West China Hospital, Sichuan University, Chengdu, Sichuan, 610041 China \\ ${ }^{2}$ Department of Anesthesiology, Cheng Du Shang Jin Nan Fu Hospital, Chengdu, Sichuan, 610000 China \\ "Li Zhou and Hong Chang contributed equally to this work
}

*Corresponding author: Chunling Jiang, Department of Anesthesiology, West China Hospital, Sichuan University, Chengdu, Sichuan, 610041 China, Tel: +8618980601096, Fax: +862885423595; E-mail: jiang_chunling@yahoo.com

\begin{abstract}
Pseudoaneurysm of mitral aortic intervalvular fibrosa (P-MAIVF) is a rare but potentially catastrophic condition. P-MAIVF is often associated with infective endocarditis, aortic valve surgery or chest trauma. However, P-MAIVF attributed to congenital abnormality is even rarer. Here, we present a case of chronic, non-infective P-MAIVF associated with congenital bicuspid aortic valve. In the present case, there is neither a history nor sign suggesting infective endocarditis, nor positive blood or tissue culture results. The preoperative Transesophageal echocardiography (TEE) examination detected P-MAIVF with severe regurgitation of bicuspid aortic valve, which was misdiagnosed as pure bicuspid aortic valve regurgitation by transthoracic echocardiogram (TTE). Consequently, TEE provides valuable information on the diagnosis and choice of surgery for the patient.
\end{abstract}

Keywords: Pseudoaneurysm of mitral aortic intervalvular fibrosa (P-MAIVF); Bicuspid aortic valve; Aortic regurgitation; Transesophageal echocardiography (TEE); Transthoracic echocardiogram (TTE)
Received date: May 22, 2017

Accepted date: June 5, 2017

Published date: June 6, 2017

Citation: Jiang, C., et al. Pseudoaneurysm of Mitral-Aortic Intervalvular Fibrosa Following Regurgitation of Congenital Bicuspid Aortic Valve in a 63-Year-Old Patient: A Case Report. (2017) J Anesth Surg 4(1): 68-70.

DOI: $10.15436 / 2377-1364.17 .077$

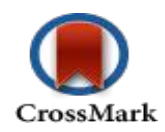

\section{Background}

Pseudoaneurysm of mitral aortic intervalvular fibrosa (P-MAIVF), located between the mitral and aortic valves, is an uncommon but potentially catastrophic condition ${ }^{[1]}$. P-MAIVF is often associated with infective endocarditis, aortic valve surgery or chest trauma ${ }^{[2,3]}$. However, congenital heart diseases, such as bicuspid aortic valve, ventricular septal defect or patent ductus arteriosus, are rare causative factors of P-MAIVF ${ }^{[4,5]}$. Here, we present a case of congenital P-MAIVF in a 63-year-old patient with severe regurgitation of bicuspid aortic valve detected by preoperative trans-oesophageal echocardiography (TEE).

\begin{abstract}
Case Presentation
A 63-year-old female patient was admitted to the hospital, with complaints of fatigue for 7 days. There was neither a history nor signs suggesting infective endocarditis, rheumatic fever, cardiac surgery, or blunt trauma to the chest in the past. On physical examination, she had a grade $3 / 6$ diastolic murmur at the left third intercostals space. The results of laboratory tests were normal. Besides, blood culture showed no positive sign of infection. A transthoracic echocardiogram (TTE) was performed, which demonstrated severe aortic regurgitation, a slightly calcified bicuspid aortic valve and moderate left ventricular dilation (left ventricular end-systolic diameter $50 \mathrm{~mm}$ ), with a normal ventricular ejection fraction $(62 \%)$. The patient was then scheduled for aortic valve replacement.
\end{abstract}


After general anesthesia, a transesophageal echocardiography (TEE) examination was performed. Unexpectedly, TEE detected a pseudoaneurysm in the region of the MAIVF, in addition to the aortic regurgitation and bicuspid aortic valve (Figure 1). Midesophegeal aortic valve long-axis view demonstrated a 1.3 $\times 0.4 \mathrm{~cm}$ echolucent area with a narrow neck lying between the anterior mitral leaflet and aortic valve (Figure 2), which was identified as systolic expansion and diastolic collapse within the area of the mitral aortic intervalvular fibrosa (MAIVF) by colour flow Doppler, with flow extending into the left ventricular outflow tract (Figure 2).

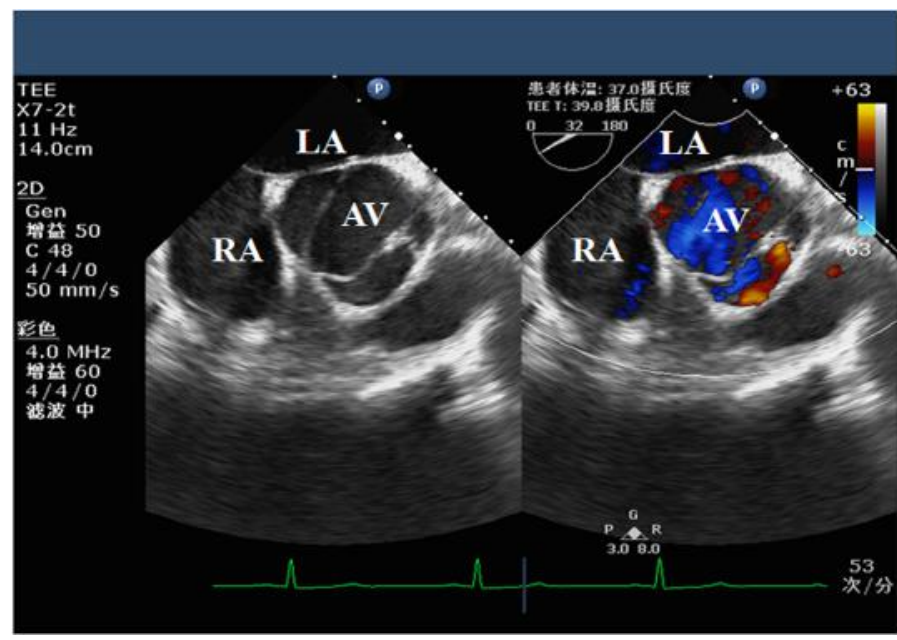

Figure 1: Midesophegeal aortic valve shor-axis view of bicuspid aortic valve. LA, left atrium; RA, right atrium; $\mathrm{AV}$, aortic valve.
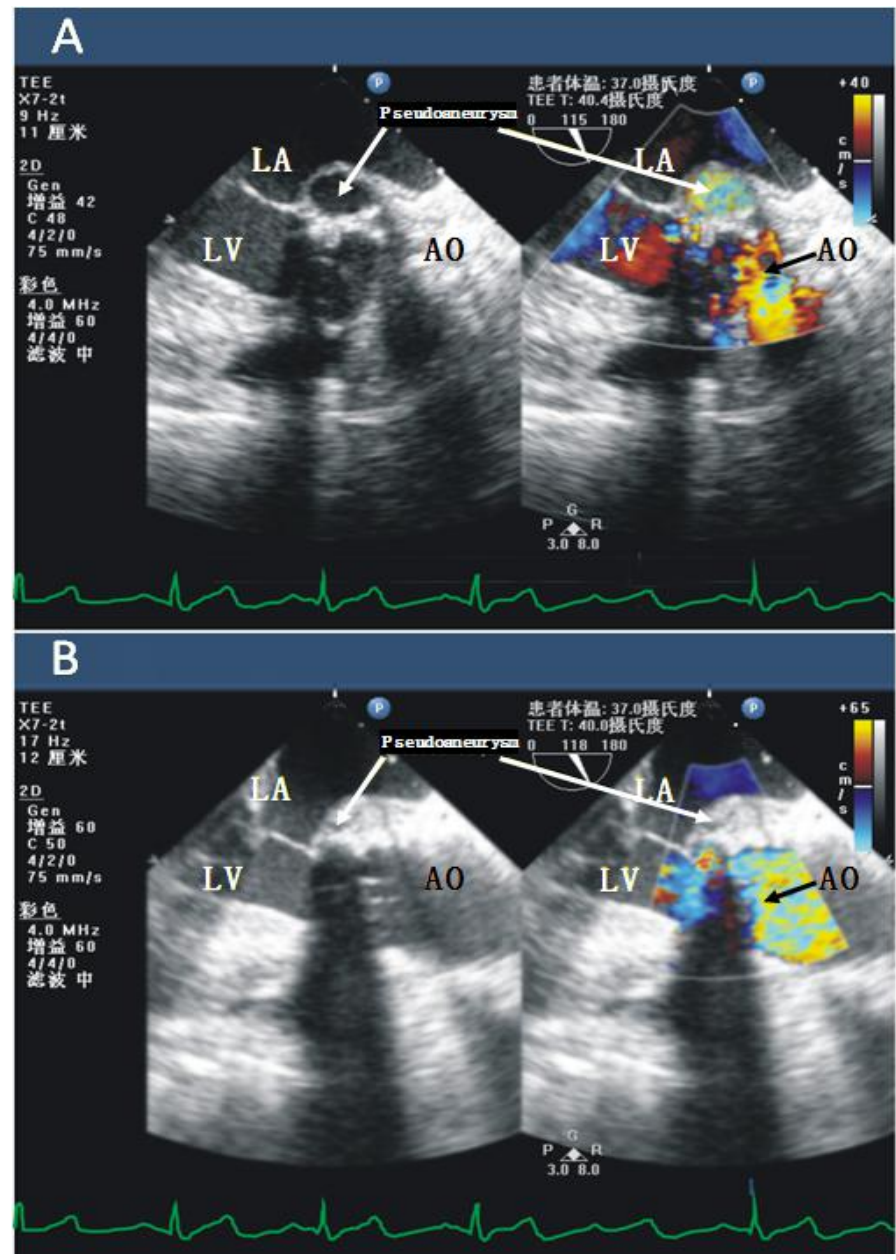

Figure 2: Midesophegeal aortic valve long-axis view of pseudoaneurysm of mitra-aortic intervalvular fibrosa. Midesophegeal aortic valve long-axis view demonstrated a $1.3 \times 0.4 \mathrm{~cm}$ echolucent area with a narrow neck lying between the anterior mitral leaflet and aortic valve, which was identified as systolic expansion and diastolic collapse within the area of the mitral-aortic intervalvular fibrosa by colour flow Doppler, with flow extending into the left ventricular outflow tract. LA, left atrium; LV, left ventricular; AO, ascending aorta.

Direct observation at surgery showed that the region of the mitral-aortic intervalvular fibrosa was destroyed, and the aneurysm orifice was located at the conjunction between the noncoronary and left coronary cusp, with slight aortic annular calcification. The surgery included replacement of aortic valve, excision of aneurysm wall and repair of the fistula connecting the left ventricular outflow tract with MAIVF. The orifice of the pseudoaneurysm was closed using a pericardial patch. Duration of the whole procedure was 235 minutes. Postoperative recovery was uneventful.

A histopathologic examination of the excised aneurysm wall revealed slightcalcification. Bacterial, mycobacterial and fungal cultures fromthe resected tissue were negative.

\section{Discussion}

The MAIVF is an membranous structure located adjacent to the left ventricular outflow tract between the aortic and mitral valve providing functional and anatomical integrity5. Since the MAIVF is relatively avascular, it becomes more prone to infection, trauma or pseudoaneurysm formation ${ }^{[5,6]}$. P-MAIVF is a rare but potentially life-threatening entity ${ }^{[1,7,8]}$. It commonly occurs in patients with infective endocarditis, aortic valve surgery, or chest trauma ${ }^{[1,5,6]}$. Few cases have been attributed to congenital abnormality, including bicuspid aortic valve, ventricular septal defect, atrial septal defect, or patent ductus $\operatorname{arteriosus}^{[4,5]}$. The pathogenesis of P-MAIVF still remains largely undefined, but it has been suggested that it may develop from either direct extension of endocarditis of aortic valve or from an "infected" aortic regurgitation jet which strikes the subvalvular structures $^{[9]}$.

Interestingly, in the present case, there is neither a history nor signs suggesting infective endocarditis, nor positive blood or tissue culture results, namely no evidence of infection. However, the congenital bicuspid aortic valve in this patient predisposed to regurgitation and pseudoaneurysm formation in the mitral-aortic intervalvular fibrosa area, likely because of congenital weaknesss in the area of MAIVF ${ }^{[10,11]}$ and regurgitation jet striking the fragile structures. Most importantly, the histopathologic examination of the excised aneurysm wall revealed severe calcification, confirming a longstanding process for pseudoaneurysm formation. Consequently, we speculate that this is a rare case of chronic, non-infective P-MAIVF associated with congenital bicuspid aortic valve.

Notably, in our case, the TEE provides valuable information on the diagnosis and choice of surgery. The preoperative Transesophageal echocardiography examination detected P-MAIVF, which was misdiagnosed as pure bicuspid aortic regurgitation by TTE. TEE, with better signal to noise ratio and proximity of probe to the heart, facilitated the visualization of this area and enhanced the detection and assessment of pseudo- 
P-MAIVF Following Regurgitation of Congenital Bicuspid Aortic Valve aneurysm $^{[3,12]}$.
In summary, pseudoaneurysm formation of the MAIF attributed to congenital bicuspid aortic valve is a rare, but known, entity. It should be kept in mind in patients who were diagnosed with bicuspid aortic valve. TEE serves as a very useful diagnostic tool and has become a more sensitive technique to evaluate this condition.

Acknowledgement: This work was supported by the Sichuan Provincial Scientific Grant (2017SZ0110) and National Natural Science Foundation of China (81600006).

\section{Reference}

1. Kassim, T.A., Lowery, R.C., Nasur, A., et al. Pseudoaneurysm of mitral-aortic intervalvular fibrosa: two case reports and review of literature. (2010) Eur J Echocardiogr 11(3): E7.

Pubmed | Crossref

2. Takimoto, K., Arai, F., Kita, T., et al. Pseudoaneurysm of the mitral-aortic intervalvular fibrosa on a native aortic valve following infective endocarditis. (2010) J Anesth 24(2): 260-263.

Pubmed | Crossref | Others

3. Afridi, I., Apostolidou, M.A., Saad, R.M., et al. Pseudoaneurysms of the mitral-aortic intervalvular fibrosa: dynamic characterization using transesophageal echocardiographic and Doppler techniques. (1995) J Am Coll Cardiol 25(1): 137-145.

Pubmed | Crossref

4. Xie, M., Li, Y., Cheng, T.O., et al. Pseudoaneurysm of the mitral-aortic intervalvular fibrosa. (2013) Int J Cardiol 166(1): 2-7.

Pubmed | Crossref

5. Sahan, E., Gul, M., Sahan, S., et al. Pseudoaneurysm of the mitral-aortic intervalvular fibrosa: A new comprehensive review. (2015) Herz 40 Suppl 2: 182-189.

Pubmed | Crossref | Others

6. Grimaldi, A., Ho, S.Y., Pozzoli, A., et al. Pseudoaneurysm of mitral-aortic intervalvular fibrosa. (2011) Interact Cardiovasc Thorac Surg 13(2): 142-147.

Pubmed | Crossref

7. Stechert, M.M., Kellermeier, J.P. Aorto-atrial fistula: an important complication of aortic prosthetic valve endocarditis. (2007) Anesth Analg 105(2): 332-333.

Pubmed | Crossref

8. Sudhakar, S., Sewani, A., Agrawal, M., et al. Pseudoaneurysm of the mitral-aortic intervalvular fibrosa (MAIVF): A comprehensive review. (2010) J Am Soc Echocardiogr 23(10): 1009-1018 quiz 1112.

Pubmed | Crossref | Others

9. Salerno, D., Donati, G., Forconi, S., et al. Giant pseudoaneurysm of the mitro-aortic intervalvular fibrosa: incidental diagnosis. (2008) Intern Emerg Med 3(3): 279-282.

Pubmed | Crossref

10. Qizilbash, A.H., Schwartz, C.J. False aneurysm of left ventricle due to perforation of mitral-aortic intervalvular fibrosa with rupture and cardiac tamponade. Rare complication of infective endocarditis. (1973) Am J Cardiol 32(1): 110-113.

Pubmed | Crossref | Others

11. Demir, M. Left ventricular systolic and diastolic function in subjects with a bicuspid aortic valve without significant valvular dysfunction. (2013) Exp Clin Cardiol 18(1): e1-4.

Pubmed

12. Karalis, D.G., Bansal, R.C., Hauck, A.J., et al. Transesophageal echocardiographic recognition of subaortic complications in aortic valve endocarditis Clinical and surgical implications. (1992) Circulation 86(2): 353-362.

Pubmed | Crossref
Ommega Online Publishers

Journal Title: Journal of Anesthesia and Surgery (JAS)

Journal Short Name: J Anesth Surg
Journal ISSN: 2377-1364

E-mail: anestheisa@ommegaonline.com

Website: www.ommegaonline.org 